\title{
Two-dimensional electronic spectroscopy as a tool for tracking molecular conformations in DNA/RNA aggregates
}

\author{
Javier Segarra-Martí, (D) *a Vishal K. Jaiswal, ${ }^{\mathrm{b}}$ Ana Julieta Pepino, ${ }^{\mathrm{b}}$ \\ Angelo Giussani, (D) ' Artur Nenov, ${ }^{\text {c Shaul Mukamel, }}{ }^{d}$ \\ Marco Garavelli (iD *b and Ivan Rivalta (iD *a
}

\section{Received 30th September 2017, Accepted 17th October 2017 \\ DOI: $10.1039 / \mathrm{c} 7 \mathrm{fd} 00201 \mathrm{~g}$}

A computational strategy to simulate two-dimensional electronic spectra (2DES) is introduced, which allows us to analyse ground state dynamics and to sample and measure different conformations attained by flexible molecular systems in solution. An explicit mixed quantum mechanics/molecular mechanics (QM/MM) approach is employed for the evaluation of the necessary electronic excited state energies and transition dipole moments. The method is applied towards a study of the highly flexible water-solvated adenine-adenine monophosphate (ApA), a system featuring two interacting adenine moieties that display various intermolecular arrangements, known to deeply affect their photochemical outcome. Molecular dynamics simulations and cluster analysis have been used to select the molecular conformations, reducing the complexity of the flexible ApA conformational space. By using our sum-over-states (SOS) approach to obtain the 2DES spectra for each of these selected conformations, we can discern spectral changes and relate them to specific nuclear arrangements: close lying $\pi$-stacked bases exhibit a splitting of their respective ${ }^{1} L_{a}$ signal traces; $T$ stacked bases exhibit the appearance of charge transfer states in the low-energy Vis probing window while displaying no ${ }^{1} L_{a}$ splitting, being particularly favoured when promoting amino to 5-ring interactions; unstacked and distant adenine moieties exhibit signals similar to those of the adenine monomer, as is expected for non-interacting nucleobases. 2DES maps reveal the spectral fingerprints associated with specific molecular conformations, and are thus a promising option to enable their quantitative spectroscopic detection beyond standard 1D pump-probe techniques. This is expected to aid the understanding of how nucleobase aggregation controls and modulates the photostability and photo-damage of extended DNA/RNA systems.

\footnotetext{
${ }^{a}$ Univ Lyon, Ens de Lyon, CNRS, Université Claude Bernard Lyon 1, Laboratoire de Chimie UMR 5182, F-69342, Lyon, France.E-mail: Javier.segarra-marti@ens-lyon.fr; ivan.rivalta@ens-lyon.fr

${ }^{b}$ Dipartimento di Chimica Industriale "Toso Montanari”, Università di Bologna, Viale del Risorgimento, IT40136, Italy. E-mail: marco.garavelli@unibo.it

'Department of Chemistry, University College London, 20 Gordon Street, London WC1H OAJ, UK

${ }^{d}$ Department of Chemistry, University of California, Irvine, California 92697-2025, USA
} 


\section{Introduction}

The conformations attained by molecular systems in condensed (liquid or solid) phases are often surrounded by controversy given the difficulties faced by theorists and experimentalists in locating and assigning specific spectroscopic fingerprints pertaining to a given molecular motif. Various molecular arrangements, which often exhibit completely different photophysical properties due to their featured intermolecular interactions, are thus key to properly assessing and rationalising certain bulk and interfacial phenomena that are of paramount interest, such as the ultrafast and efficient electronic energy transfer processes registered in light harvesting complexes or the specific charge/transport mechanisms behind state-of-the-art solar cells. ${ }^{1}$ This is especially true in biomolecular systems, where a specific conformation may impair or even completely alter a concrete cellular function. One particularly important case that highlights this effect would be the photostability of genetic material, ${ }^{2,3}$ and how the photophysics of DNA/RNA sequences are severely affected in dimeric and multimeric species by the presence of additional neighbouring stacked nucleobases within a given strand in the double helix. Nucleobase stacking might indeed increase their excited state lifetime and potentially make them more prone to photochemical mutations, ${ }^{4-7}$ which is in contrast to the efficient and ultrafast decay registered in isolated nucleobases, ${ }^{\mathbf{8} 9}$ which is expected to strongly promote its photoprotection mechanisms. ${ }^{10}$ This effect, which is believed to strongly modulate the complexity of DNA photophysics, ${ }^{11,12}$ is particularly hard to measure using standard pump-probe set-ups, which are the experimental workhorse in photochemistry. In standard 1D pump-probe spectroscopy, different signals from diverse molecular motifs overlap strongly in space and time, leading to highly congested spectral regions whose complex interpretation remains elusive.

A recently developed technique that can overcome these aforementioned limitations is two-dimensional (2D) electronic spectroscopy (2DES), which is able to disentangle signals arising from different intermolecular interactions that remain hidden in 1D pump-probe spectra. In these multi-pulse techniques, which are frequently used in the $\mathrm{IR}^{\mathbf{1 3 , 1 4}}$ and which have been recently extended to the electronic regime, ${ }^{15-17}$ the system is excited by a sequence of three pulses with controlled delays, $t_{1}$ (coherence time) and $t_{2}$ (population/waiting time), with the third pulse at time $t_{3}$ emitting the third-order nonlinear signal $\mathrm{S}\left(t_{1}, t_{2}, t_{3}\right) \cdot{ }^{18-21} \mathrm{By}$ carrying out Fourier transform with respect to $t_{1}$ and $t_{3}$, one can extract a sequence of $2 \mathrm{D}$ maps $\mathrm{S}\left(\Omega_{1}, t_{2}, \Omega_{3}\right)$ for different waiting times, $t_{2}$. These 2D maps contain a vast number of signals that can potentially be employed for tracking all sorts of localised, delocalised, energy and charge transfer photoinduced phenomena with unprecedented detail. However, their interpretation proves to be challenging. It is in this context that theoretical chemistry comes in handy; by simulating the spectra we can separate the different peaks into their contributing specific electronic transitions, thus providing a route map to recognise, understand and interpret the main fingerprints observed..$^{22,23}$

In this contribution we introduce a computational strategy that combines ground-state molecular dynamics with $\mathrm{QM} / \mathrm{MM}$ approaches in order to sample the conformational space of dimeric and multimeric flexible bio-chromophores and separate their different nuclear arrangements to be characterised 
spectroscopically by 2DES. The methodology is applied to ApA, a model system that contains both unstacked (non-interacting) and $\pi$ - or T-stacked (interacting) adenine moieties, and is shown to display distinct fingerprints for these different conformations, thus proving to be a promising tool to separate them and understand precisely how they affect the underlying photophysical and photochemical pathways in such complex and extended DNA/RNA systems.

\section{Computational details}

Molecular dynamics simulations were carried out using the AMBER 11 software package. $^{24,25}$ A $12 \times 12 \times 12 \AA$ cubic box was employed making use of the parmbsc0 (ref. 26) and TIP3 $\mathrm{P}^{27}$ force fields for the ApA moiety and water molecules, respectively. A 100 ns trajectory was carried out, extracting one snapshot every 2.5 ps, totalling 40000 frames. Root mean square deviation (RMSD) cluster analysis was carried out on those 40000 structures and was set within $1.5 \AA$, extracting 89 different clusters. From these, 17 were retained for further electronic structure theory evaluations, accounting for up to $\sim 75 \%$ of the conformational space sampled by the highly flexible ApA system. The selected snapshots, which are the closest structures to each centroid, were subsequently optimised by employing a complete active space self-consistent field (CASSCF) comprising 8 electrons in 8 orbitals, $4 \pi$ occupied and $4 \pi^{*}$ unoccupied, constraining phosphate and sugar groups and allowing relaxations only on the adenine moieties in order to obtain more accurate bond length distances than those given by the force field. This has been done due to the strong dependence of the high-energy excited state manifold on the bond length distances displayed by adenine, as has been shown elsewhere. ${ }^{28}$

All electronic structure computations were carried out using the MOLCAS 8 package. ${ }^{29}$ The restricted active space self-consistent field (RASSCF) ${ }^{30}$ and its second-order perturbation theory extension (RASPT2) ${ }^{31,32}$ were applied to the aforementioned selected structures. Cholesky decomposition was used to speedup the two-electron integrals ${ }^{33-35}$ and in conjunction with Atomic Natural Orbital $(\mathrm{ANO})^{36}$ large-type basis sets with valence double- $\zeta$ polarised contractions. Only the adenine nucleobases were included in the quantum mechanical region, with atom links connecting the QM (adenine moieties) and MM (phosphate and water molecules) layers. ${ }^{37}$ An active space of 24 electrons in 18 orbitals was employed, comprising the whole $\pi$ occupied valence space of both adenine moieties and all except the highest $\pi^{*}$ virtual orbital of each moiety. Up to four holes/electrons were included in the RAS1/RAS3 subspaces, respectively, leading to RASPT2 $(4,12|0,0| 4,6)$, and these were averaged with equal weights over 100 states. RASPT2 $(4,6|0,0| 4,3)$ was used for the monomer as previously benchmarked ${ }^{28}$ and averaged over 25 states, with its active space being duplicated for the dimer. This particular active space was used considering previous benchmark studies where its adequacy to represent the energies and transition dipole moments with respect to reference RASPT2 $(0,0|12,10| 2,12)$ computations on the adenine monomer was assessed. ${ }^{28}$ An imaginary level shift ${ }^{38}$ of 0.6 a.u. was employed for the dimer, with 0.5 a.u. employed for the monomer as previously benchmarked, ${ }^{28}$ and with the IPEA shift being set to zero. ${ }^{39,40}$ The restricted active space state interaction (RASSI) method ${ }^{41,42}$ was employed to obtain the required transition dipole moments among the different electronic ground and excited states. 
The resulting energy levels and transition dipole moments were then used in conjunction with the Sum-Over-States (SOS) approach ${ }^{43}$ within the dipole approximation and a QM/MM scheme (SOS//QM/MM) ${ }^{18}$ to simulate the 2DES as implemented in the Spectron 2.7 program. ${ }^{4}$ Further details on the working equations have been given elsewhere. ${ }^{28}$ Spectral lineshapes were simulated assuming that the dephasing is caused by pure dephasing in the Markovian approximation. Effectively, the spectra of the individual snapshots are subject to a constant line broadening of $1000 \mathrm{~cm}^{-1}$, which has been set so that the broadenings along $\Omega_{1}$ for the total ApA spectrum mimic the experimental broadening recently measured in 2DUV experiments for adenine. ${ }^{17}$ Although several computational approaches have been devised recently to consider spectral diffusion and non-adiabatic effects on the simulated 2DES, ${ }^{45-49}$ the size of the current system prevents the usage of more sophisticated approaches. Quasi-absorptive signals were simulated for the condition of a collinear pump pulse pair (thus simultaneously recording rephrasing and non-rephasing contributions). All reported signals used the all-parallel $x x x x$ pulse polarisation configuration, were plotted on a linear scale and considered white light (infinite broadband) pulses. This allowed us to explore the variety of signals that may be present in the 2D spectra. Both ground state bleaching (GSB) and stimulated emission (SE) contributions appear as negative (blue) peaks, whereas excited-state absorptions (ESAs) appear as positive (red) peaks in the 2D spectra.

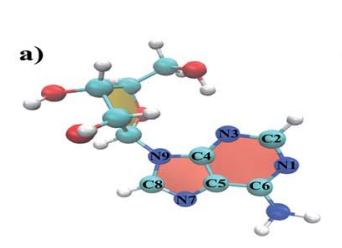

b)

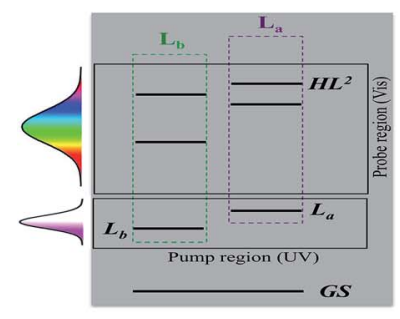

c)
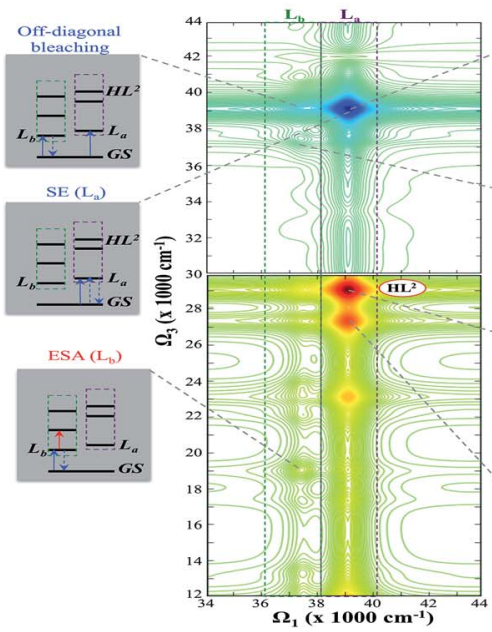

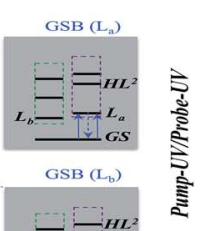

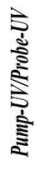

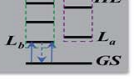

ESA $\left(L_{a}\right)$

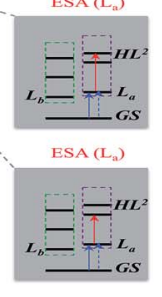

Fig. 1 2DES spectrum of adenosine monophosphate, where (a) displays the molecular system and atom labelling, (b) shows a scheme of the energy levels that are accessible with different laser pulses and (c) shows the 2DES in the Vis (lower panel) and UV (upper panel) probe windows, together with energy level schemes depicting the different signals featured in terms of the laser pulse sequence. A red colour denotes positive (ESA) signals. A blue colour denotes negative (GSB, off-diagonal bleach and SE) signals. The SE signal associated with the ${ }^{1} L_{b}$ state was not included in the scheme for simplicity, being placed on top of $G S B\left(L_{b}\right)$. The signal intensity in each spectrum is normalised according to the absolute value of the most intense signal. 


\section{Results}

The 2DES of water-solvated adenosine is considered first (see Fig. 1) and is then used as a reference in order to interpret the signals arising in the ApA dimer. As shown in Fig. 1a, adenosine features a sugar group connected to the nucleobase through the N9 position, analogous to the adenine moieties contained in ApA, and is expected to exhibit mostly the same spectral signals as those of adenine while not exhibiting the known mix of tautomers $(7 \mathrm{H}$-and $9 \mathrm{H}$-adenine) in the bare nucleobase embodying different excited state lifetimes ${ }^{50,51}$ that complicate its analysis. As can be seen in Fig. 1b, by pumping (photo-exciting) in the absorbing UV region, the two main excited states of $\pi \pi^{*}$ character are expected to be populated, with a more stable and less intense ${ }^{1} \mathrm{~L}_{\mathrm{b}}$ state and a more intense and higher in energy ${ }^{1} \mathrm{~L}_{\mathrm{a}}$ state, even though their relative energetic state ordering is still under debate. ${ }^{\mathbf{2 8 , 5 2 , 5 3}}$ These two neighbouring excited states that are accessible from the ground state give rise to two traces along $\Omega_{1}$ in the 2DES spectra, featuring ESAs in the Vis probing window and GSB and off-diagonal bleaching signals in the UV. This strong GSB contribution mostly obscures all signals from ESAs in the UV window. Fig. 1c shows the spectra together with a simple scheme depicting the pulse sequence leading to each of the signals, with the arrows representing the electronic transitions produced by the multi-pulse laser sequence. GSB arises from an excitation from the ground state to a given excited state, de-excitation from that state back to the ground state and again an excitation to the very same excited state. Off-diagonal bleaching follows the same first two excitations as GSB, with the third pulse promoting excitation to another excited state. SE and ESA signals are formed from a two-pulse coherent excitation from the ground state to a given excited state, which leads to population of the excited state, from which the third pulse either promotes excitation to higher lying states (ESA) or emission back to the ground state (SE).

Our results for water-solvated adenosine were compared against previous reference computations on gas-phase adenine performed with larger active spaces and basis sets in vacuo, ${ }^{28}$ with notable differences observed along $\Omega_{1}$ (linear absorption of the photo-activated ${ }^{1} \mathrm{~L}_{\mathrm{a}}$ and ${ }^{1} \mathrm{~L}_{\mathrm{b}}$ states of adenine) which is redshifted by $0.2-0.3 \mathrm{eV}$, which is partially expected due to the addition of a polar protic solvent such as water that produces an analogous red-shifting effect in DNA/RNA nucleobases. ${ }^{37,54-56}$ It is worth noting that more accurate approaches could be used to compute the spectrum of the monomer, as shown in previous benchmarks, ${ }^{28}$ yet those cannot be extended to dimeric species, thus justifying our use of a cost-effective RASPT2 $(4,6|0,0| 4,3)$ scheme. Beyond the red-shift observed along $\Omega_{1}$, the other main difference observed with respect to the reference is the relative intensity of the ${ }^{1} \mathrm{~L}_{\mathrm{b}}$ trace with respect to that of ${ }^{1} \mathrm{~L}_{\mathrm{a}}$, which appears to be obscured due to the usage of infinitely wide pulses that enhance the ${ }^{1} \mathrm{~L}_{\mathrm{a}}$ trace, as opposed to the tailored finite pulses employed in the reference computations to highlight this relatively dark state. ${ }^{28}$ The absence of signals in the low-energy window as opposed to the reference computations ${ }^{28}$ can be attributed to the use of infinitely broad pulses, which obscure them as has also been reported for the ${ }^{1} \mathrm{~L}_{\mathrm{b}}$ trace. The usage of such pulses allows us to focus on the overall most intense signals without considering particular pump and probe setups that could potentially be used with finite pulses to enhance specific peaks, 
which is for the moment out of scope of the present manuscript. Our reduced RASPT2 $(4,6|0,0| 4,3)$ model is able to quantitatively reproduce the most important signal, that is, the one relating to the transition between ${ }^{1} \mathrm{~L}_{\mathrm{a}}$ and the doubly excited HOMO to LUMO state placed $\sim 29000 \mathrm{~cm}^{-1}$ along $\Omega_{3}\left(\mathrm{HL}^{2}\right)$, which is very intense and appears in different spectral regions for the different nucleobases, ${ }^{28,57}$ thus being the main monomer-based fingerprint to potentially follow excited state decays on separate monomers simultaneously. An additional signal right below $\mathrm{HL}^{2}$ can be observed in the spectrum (see Fig. 1c), which relates to a $\pi \pi^{*} \leftarrow{ }^{1} \mathrm{~L}_{\mathrm{a}}$ transition that appears to be less intense and that, given that additional homogeneous broadenings were included, would most likely merge with that of $\mathrm{HL}^{2}$, with its in-depth analysis being considered not as important. In the higher energy UV-probe window all signals are obscured in solution, as opposed to what was observed in the gas phase reference computations, ${ }^{28}$ where ESAs along both the ${ }^{1} \mathrm{~L}_{\mathrm{b}}$ and ${ }^{1} \mathrm{~L}_{\mathrm{a}}$ traces could still be observed, albeit at low intensity compared to the GSB signals.

As indicated previously, in order to obtain a 2DES spectrum that represents the whole conformational space, a reductionist approach was employed whereby cluster analysis was performed and the most populated clusters along the dynamics were used to reduce the number of QM/MM evaluations, which is the actual bottleneck of the computational procedure. In this way, by considering the first 17 clusters (from the most to the least populated), which have been labelled in alphabetical (from A to Q) order, we can account for up to $\sim 75 \%$ of the sampled conformational space of ApA. All of the details are given in Table 1, which contains the concrete number of structures contained in each cluster, their contribution to the overall conformational space, a normalised estimate

Table 1 Clustering parameters obtained from the 100 ns molecular dynamics simulation of ApA. Relative weights are normalised and fitted to \%o for the 2DES simulations. C5-C5 distance ranges are given (in $\AA$ ) for each cluster.

\begin{tabular}{|c|c|c|c|c|c|}
\hline Cluster & $\begin{array}{l}\text { No. of } \\
\text { snapshots } \\
\text { contained }\end{array}$ & $\begin{array}{l}\% \text { over } \\
\text { total (40 } 000 \\
\text { snapshots) }\end{array}$ & $\begin{array}{l}\text { Normalised \% } \\
\text { (over } 29421 \\
\text { snapshots) }\end{array}$ & $\begin{array}{l}\text { Weight } \\
(\%)\end{array}$ & $\begin{array}{l}\text { C5-C5 } \\
\text { distance } \\
(\AA)\end{array}$ \\
\hline A & 4063 & 10.16 & 13.81 & 138 & $>6.0$ \\
\hline B & 3542 & 8.86 & 12.04 & 120 & $4.0-4.5$ \\
\hline $\mathrm{C}$ & 3470 & 8.68 & 11.79 & 118 & $<4.0$ \\
\hline $\mathrm{D}$ & 1916 & 4.79 & 6.51 & 65 & $<4.0$ \\
\hline $\mathrm{E}$ & 1744 & 4.36 & 5.93 & 59 & $4.5-5.0$ \\
\hline $\mathrm{F}$ & 1713 & 4.28 & 5.82 & 58 & $4.5-5.0$ \\
\hline G & 1689 & 4.22 & 5.74 & 57 & $4.0-4.5$ \\
\hline $\mathrm{H}$ & 1681 & 4.20 & 5.71 & 57 & $5.0-6.0$ \\
\hline I & 1661 & 4.15 & 5.65 & 57 & $4.5-5.0$ \\
\hline $\mathrm{J}$ & 1498 & 3.75 & 5.09 & 51 & $>6.0$ \\
\hline K & 1496 & 3.74 & 5.08 & 51 & $<4.0$ \\
\hline $\mathrm{L}$ & 1245 & 3.11 & 4.23 & 42 & $4.5-5.0$ \\
\hline M & 917 & 2.29 & 3.12 & 31 & $>6.0$ \\
\hline $\mathrm{N}$ & 821 & 2.05 & 2.79 & 28 & $<4.0$ \\
\hline $\mathrm{O}$ & 793 & 1.98 & 2.70 & 27 & $5.0-6.0$ \\
\hline $\mathrm{P}$ & 601 & 1.50 & 2.04 & 20 & $4.0-4.5$ \\
\hline $\mathrm{Q}$ & 571 & 1.43 & 1.94 & 19 & $5.0-6.0$ \\
\hline Total & 29421 & $73.55 \%$ & $99.99 \%$ & 998 & \\
\hline
\end{tabular}


(assuming sampling of the full conformational space) and the corresponding weights (in \%o) employed in the simulation of the total 2DES. As shown in Table 1, populations drop quickly in weight, with the first 8 clusters accounting for $\sim 50 \%$ of the population while the next 9 represent $\sim 25 \%$ of the conformational space; cluster A accounts for 7 times the contribution of cluster Q. The $\sim 75 \%$ conformational space considered is assumed to be made into the full space for obtaining the concrete weighting factors to be used for each of the clusters, thus accounting for their different contributions to the overall 2DES of ApA. At this point it is important to note that a more accurate clustering method than the RMSD adopted here would be desirable in order to obtain less clusters yet be more representative, thus further minimising the number of $\mathrm{QM} / \mathrm{MM}$ evaluations required and provide better weightings for the simulation of the full 2DES spectrum. Accurate weightings leading to a direct comparison with future experiments are beyond the scope of this work, which focuses instead on setting a viable strategy to simulate 2DES spectra and the inhomogeneous broadenings due to different molecular conformations. We have opted, indeed, for a qualitative treatment where the length of the ground state molecular dynamics simulation might not be enough to sample the full conformational space of ApA. Moreover, the force field employed was parameterised for double helix DNA chains (and not strictly for flexible systems like ApA), which may introduce an additional source of error. However, by employing the so-called "stacking coordinate" introduced by Florián and co-workers, ${ }^{58}$ we obtain a $\sim 45 \%$ population within a stacking coordinate of 4.2 , which, assuming it is representative of stacked instances, seems to be in agreement with reported circular dichroism ApA estimates and is attributed to these interacting arrangements. ${ }^{7}$ Considering all the caveats, we envision that this computational strategy could be systematically improved in various ways by employing, for instance, longer simulations, more suitable force fields, more accurate clustering techniques and ab initio simulations as well as more sophisticated treatments for the spectral line shapes.

Fig. 2 shows the molecular structure of water-solvated ApA, and both pumpUV/probe-Vis and pump-UV/probe-UV 2DES windows. The key fingerprint in the Vis probing window is the $\mathrm{HL}^{2}$ transition that was described previously, which features as the main fingerprint of each of the adenine monomers, with a less localised monomer transition displayed right below $\mathrm{HL}^{2}$ at $\sim 25 \mathrm{k} \mathrm{cm}^{-1}$ that is expected to form part of the same peak. The main difference from the monomer is the large broadening that is obtained by considering several structures, which is partially induced due to the interaction between the ${ }^{1} \mathrm{~L}_{\mathrm{a}}$ states of the different adenine moieties, thus leading to a splitting in their energy estimates along $\Omega_{1}$, as will be analysed in detail below. Further considerations would have to be made in order to properly account for the signal line shape, which is here accounted for by homogeneous broadening due to pure dephasing and is thus symmetric in the single snapshots, with asymmetries in the total spectrum arising due to the averaging of the peaks over many different conformational arrangements. The other difference that is easily observed upon comparison with the monomer is the appearance of a signal in the low-energy Vis window $\left(\sim 15 \mathrm{k} \mathrm{cm}^{-1}\right.$ along $\left.\Omega_{3}\right)$, which is attributed mainly to contributions that arise from CT states, as demonstrated by looking at their specific wave function contributions in our model. This signal is covered in some cases by localised monomer excitations that can also be observed at $\sim 13000$ and $\sim 19000 \mathrm{~cm}^{-1}$ and which obscure the CT signals, thus 

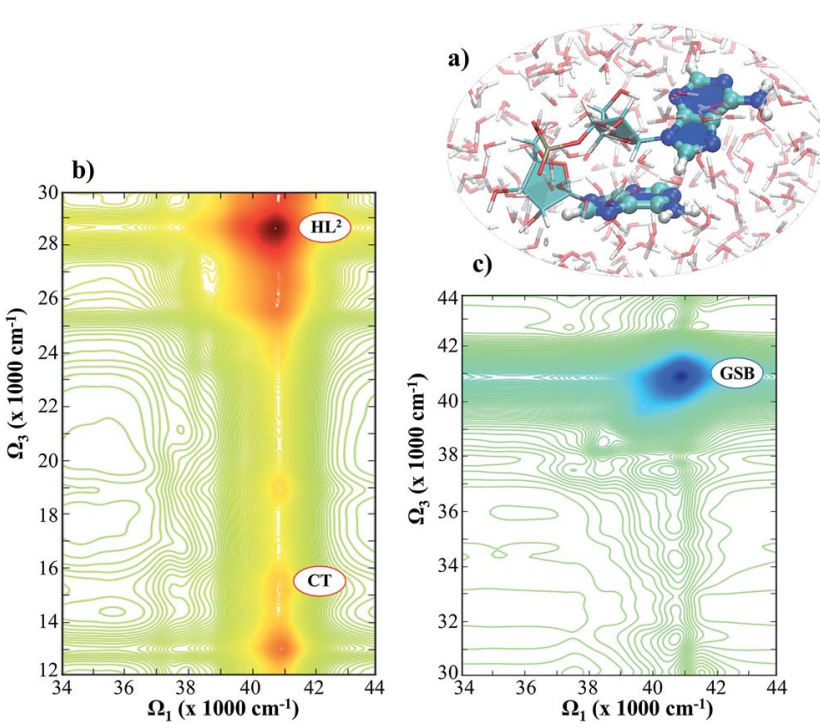

Fig. 2 2DES of water-solvated ApA considering the full conformational space (see Table 1), showing (a) the molecular structure, (b) the pump-UV/probe-Vis spectrum and (c) the pump-UV/probe-UV spectrum. The colour scheme for the 2DES spectra is set according to Fig. 1.

making their experimental characterisation challenging. These signals are not further discussed since they are associated with localised monomeric fingerprints. Moving onto the pump-UV/probe-UV window (Fig. 2c), all that can be seen is the inhomogeneous broadening of the GSB signal along the diagonal and antidiagonal widths, which arises mainly due to the different nuclear arrangements considered and which also weakly displays the contributions of the ${ }^{1} \mathrm{~L}_{\mathrm{b}}$ state diagonal GSB located at $\Omega_{1}=\Omega_{3} \approx 38000 \mathrm{~cm}^{-1}$. It is worth noting that the antidiagonal width of the signal has been reported to hold information regarding the formation of aggregates, ${ }^{59,60}$ species that are formed upon light absorption due to exciton delocalisation over several monomers that can be monitored through this particular parameter. The diverse water arrangements around the different clusters will also contribute to this broadening, with its concrete role being analysed in detail on a monomer for simplicity in an upcoming work and which is therefore out of scope of the present manuscript.

Analysis of the 2DES spectra of highly flexible systems like ApA is rather cumbersome as many of the fingerprints are specific to certain conformations. Our computational strategy allows us to dissect the full spectrum into the different clusters or representative nuclear arrangements giving rise to these signals, thus enabling us to extract the specific interactions behind a given contribution. In order to do so, we have separated the different clusters depending on their C5-C5 distance (see Fig. 1 for atom labelling), which is close to the centre of mass of the molecule and is thus a decent descriptor to group the different structures. Nevertheless, we are aware that not only the distance but also the specific orientation would play a role, which may also be said of the particular orientation of the adenine moieties with respect to the sugar groups to which they 
are attached. Thus, we use the simplest descriptor (C5-C5 distance) to partition the clusters into groups, while discussing specific nucleobase arrangements for each group of clusters.

The first group that is analysed is composed of clusters with C5-C5 distances of up to $4 \AA$, which are close and thus correspond to different $\pi$-stacked arrangements with both near face-to-face and various degrees of rotated stacked instances. Due to the lack of fingerprints in the pump-UV/probe-UV window beyond the GSB and off-diagonal bleaching, in the following discussion we will only consider the signal-rich pump-UV/probe-Vis region. Fig. 3 displays clusters C, D, K and N (see Table 1), which possess different rotated and $\pi$-stacked adenine moieties. These types of structures account for $\sim 19 \%$ of the conformational space and, importantly, do not possess fully (or perfectly) stacked moieties, which is similar to how the bases appear rotated when embedded in a double helix DNA chain. ${ }^{61,62}$ As can be seen in Fig. 3, these bases exhibit strong contributions of the doubly excited $\mathrm{HL}^{2}$ signal in the $26-30 \mathrm{k} \mathrm{cm}^{-1}$ range, oscillating along $\Omega_{1}$ and also along $\Omega_{3}$. This is more obvious in cluster $\mathrm{K}$, where the ${ }^{1} \mathrm{~L}_{\mathrm{a}}$ traces (consisting of two close-lying traces due to the ${ }^{1} \mathrm{~L}_{\mathrm{a}}$ states of the two adenine moieties) along $\Omega_{1}$ are slightly split but strongly red-shifted with respect to the other clusters due to the presence of close-lying water molecules pointing to the C4-C5 bond that are not featured in the other conformations, which seems to strongly red-shift these excitations. Despite treating the neighbouring water molecules at the MM level, their inclusion at this lower level has recently been shown for related systems to reproduce qualitative features of the low-lying electronic excitations, ${ }^{63}$ such as ${ }^{1} \mathrm{~L}_{\mathrm{a}}$, and is expected to qualitatively reproduce the solvatochromic shift for the states in the high-energy window like $\mathrm{HL}^{2}$.

The splitting of the two ${ }^{1} \mathrm{~L}_{\mathrm{a}}$ traces appears to be the main fingerprint of these nuclear arrangements, where the bases are close and strongly interact leading to
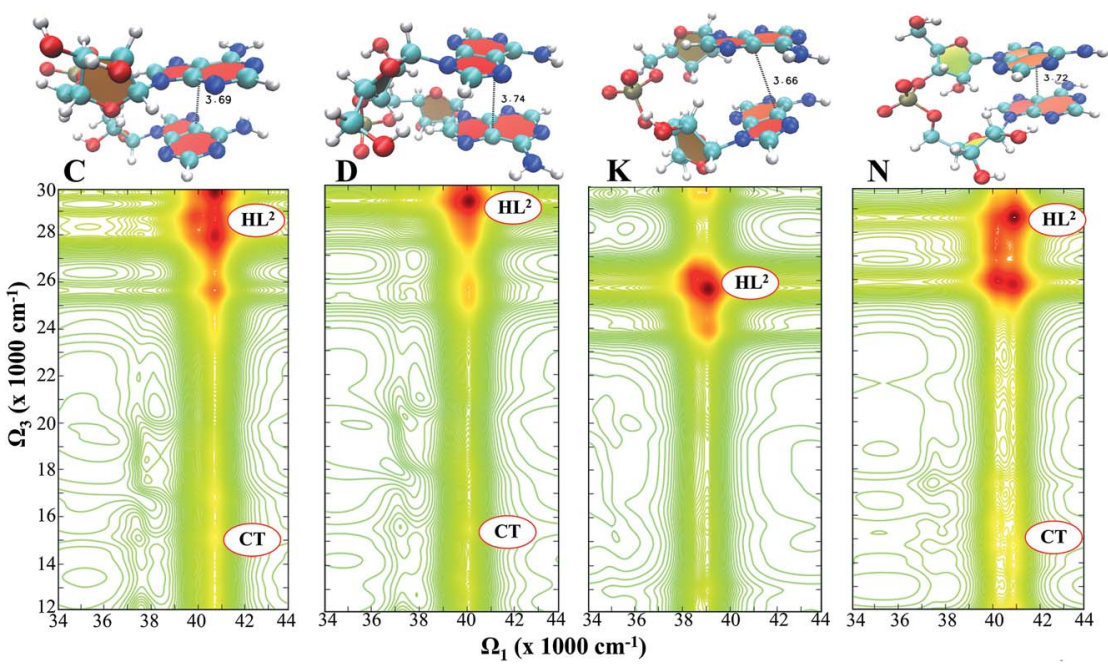

Fig. 3 2DES of the clusters with a C5-C5 distance of under $4 \AA$ A: C, D, K and N (see Table 1). The C5-C5 distances are shown on the structures. $\mathrm{HL}^{2}$ and $\mathrm{CT}$ labels are shown to aid in the assignment. The colour scheme for the 2DES spectra is set according to Fig. 1. 
a $500-1500 \mathrm{~cm}^{-1}$ splitting between these two analogous states centred at the different adenine moieties, one being red-shifted and one blue-shifted. Moreover, this fingerprint occurs along $\Omega_{1}$, which is not accessible using standard pumpprobe set-ups, therefore highlighting the potential of 2DES for disentangling conformations even for difficult cases such as homo-dimers. Besides the $\mathrm{HL}^{2}$ signal, other $\pi \pi^{*}$ states are accessed within $2000-3000 \mathrm{~cm}^{-1}$ of the latter, which are expected to merge and broaden that signal further. Additional signals appear in the low-energy window, with some of them being related to localised ESAs that were obscured in the monomer but which appeared in our previous gas-phase reference computations, ${ }^{28}$ and with some (highlighted in Fig. 3) corresponding to CT states that arise due to the proximity between adenine moieties. However, these CT states appear to be quite weak and are hard to discern from the localised ESAs on the different monomers $\left(\sim 13000 \mathrm{~cm}^{-1}\right.$ along $\left.\Omega_{3}\right)$, with the more intense contributions observed for C, D and N (Fig. 3) at $\sim 15000 \mathrm{~cm}^{-1}$.

Fig. 4 displays the clusters with C5-C5 distances of between 4 and $4.5 \AA$, namely clusters B, G and P (see Table 1 ). These account for $\sim 17 \%$ of the overall conformational space that was sampled, and exhibit a range of $\pi$-stacked and tilted geometries, which resemble those previously shown in Fig. 3. Again, the main fingerprints observed are associated with the very intense $\mathrm{HL}^{2} \mathrm{ESA}$, which is present between $25-28 \mathrm{k} \mathrm{cm}^{-1}$ along $\Omega_{3}$, red-shifted in comparison with the clusters possessing shorter C5-C5 distances, and which is strongly modulated by the position of the ${ }^{1} \mathrm{~L}_{\mathrm{a}}$ traces along $\Omega_{1}$, which oscillates as previously discussed. A splitting of the ${ }^{1} \mathrm{~L}_{\mathrm{a}}$ traces can also be observed, particularly in cluster $\mathrm{G}$, but can also be observed to a minor extent in the others. Additional $\pi \pi^{*}$ ESAs either slightly above or below the $\mathrm{HL}^{2}$ signal are again exhibited, with this peak being found (and labelled as signal 11) in reference computations, ${ }^{28}$ which refers to a HOMO-2 to LUMO+2 transition accessed through the ${ }^{1} \mathrm{~L}_{\mathrm{a}}$ state. Less intense CT
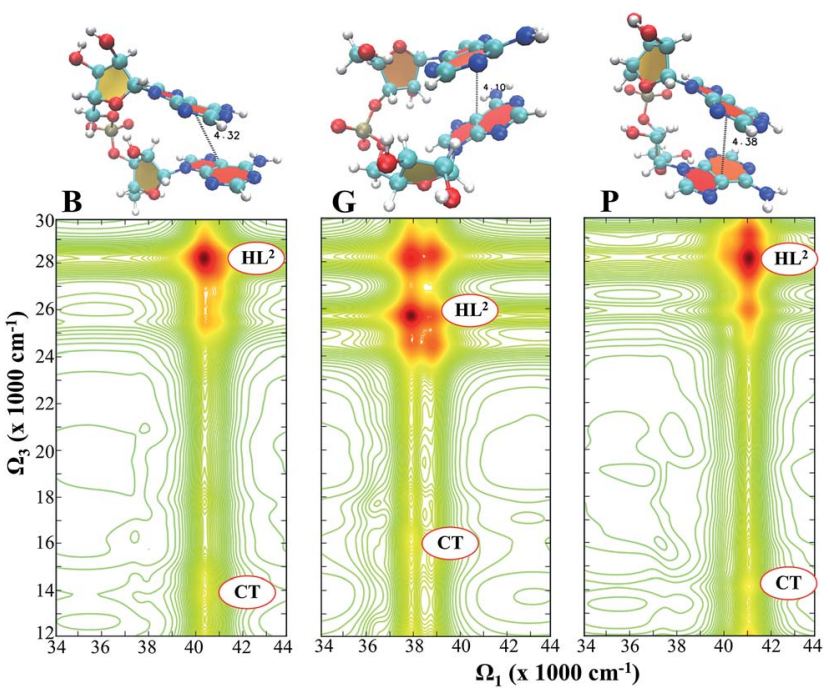

Fig. 4 2DES of the clusters with a C5-C5 distance of between 4 and $4.5 \AA$ : B, G, and P (see Table 1). The $\mathrm{C} 5-\mathrm{C} 5$ distances are shown on the structures. $\mathrm{HL}^{2}$ and $\mathrm{CT}$ labels are shown to aid in the assignment. The colour scheme for the 2DES spectra is set according to Fig. 1. 
and localised ESAs appear in the low-energy region $\left(\sim 15000\right.$ and $\sim 13000 \mathrm{~cm}^{-1}$, respectively) in comparison with those observed at shorter C5-C5 distances (see Fig. 3).

At longer C5-C5 distances, between 4.5 and $5 \AA$ A, the four clusters E, F, I and L are featured, which contribute to $\sim 16 \%$ of the full conformational space of ApA. As can be seen in Fig. 5, upon increasing the C5-C5 distance the traces of the ${ }^{1} \mathrm{~L}_{\mathrm{a}}$ states of the different adenine moieties overlap with one another, giving rise to a narrower signal along $\Omega_{1}$ that is analogous to that of the monomer and producing the main intense $\mathrm{HL}^{2}$ ESA signal at the same energies $\left(\sim 29000 \mathrm{~cm}^{-1}\right)$ as that of the monomer. An analogous set of signals is produced by both adenine moieties, which overlap displaying the other relatively intense $\pi \pi^{*}$ ESAs at slightly different energies and thus giving rise to what appears to be more signals, as can be seen in cluster $\mathrm{F}$. Due to the larger distance, the interaction among ${ }^{1} \mathrm{~L}_{\mathrm{a}}$ traces is not observed and the additional broadening along $\Omega_{1}$ is lost, thus demonstrating their non-interactive (unstacked) nature, which also exhibits no intense contributions in the low-energy Vis window $\left(12-15 \mathrm{k} \mathrm{cm}^{-1}\right)$ as was observed in the monomer. However, it is worth noting that slightly intense ESAs with CT character may still be observed in conformations displaying these long C5-C5 distances, as can be seen for clusters I and L in Fig. 5. The former presents a tilted base pointing towards the other with its amino group, which appears to enhance the appearance of CT states, whereas the latter, despite the long distances displayed, possesses a fully face-to-face stacked conformation that is expected to enhance the $\pi$-stacking interaction thus facilitating the appearance of these barely bright CT states in the low-energy Vis window, as reported above.

Fig. 6 shows C5-C5 distances of between 5 and $6 \AA$, comprising the clusters $H$, $\mathrm{O}$ and $\mathrm{Q}$ that amount to $\sim 9 \%$ of the total conformational space, showing different arrangements of distant adenine moieties that do not interact strongly with one
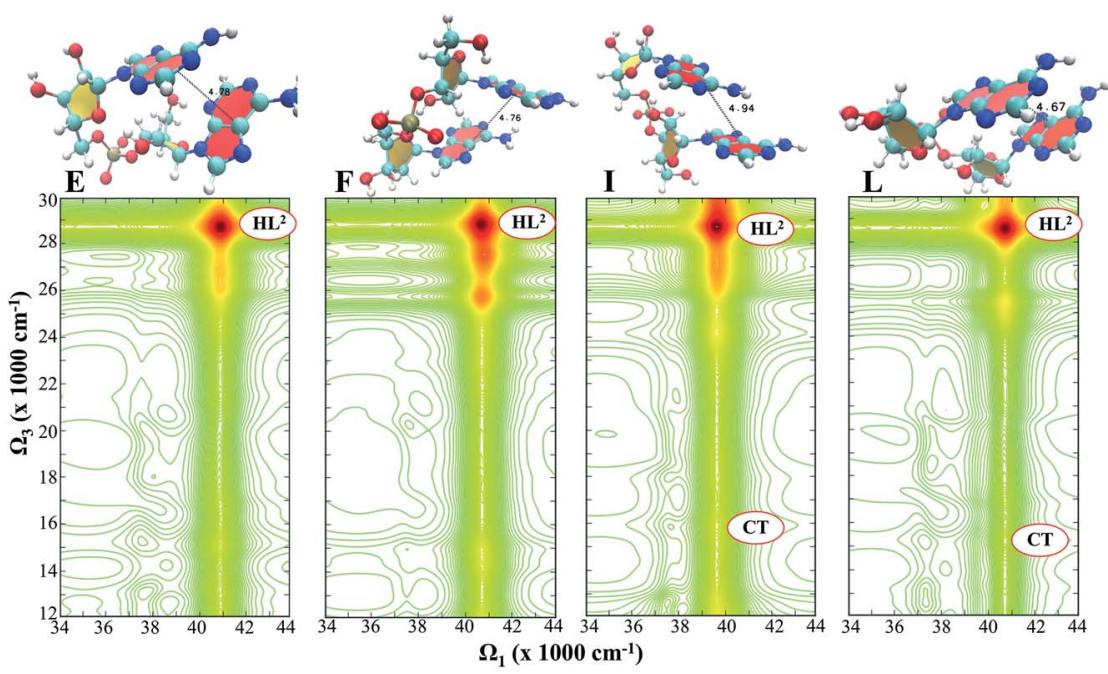

Fig. 5 2DES of the clusters with a C5-C5 distance of between 4.5 and $5 \AA$ : E, F, I and L (see Table 1). The $\mathrm{C} 5-\mathrm{C} 5$ distances are shown on the structures. $\mathrm{HL}^{2}$ and $\mathrm{CT}$ labels are shown to aid in the assignment. The colour scheme for the 2DES spectra is set according to Fig. 1. 


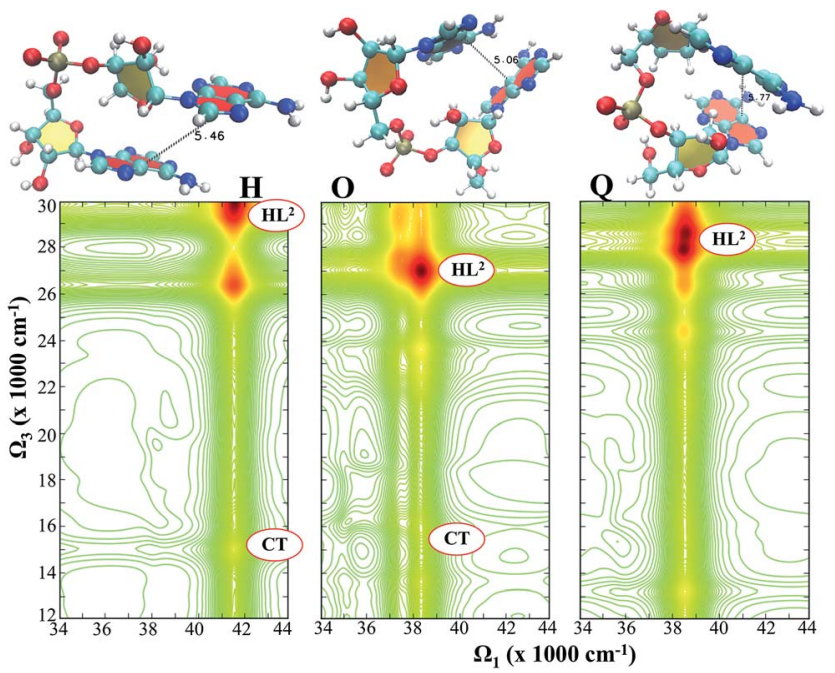

Fig. 6 2DES of the clusters with a C5-C5 distance of between 5 and $6 \AA$ : $H$, O, and $Q$ (see Table 1). The C5-C5 distances are shown on the structures. $\mathrm{HL}^{2}$ and $\mathrm{CT}$ labels are shown to aid in the assignment. The colour scheme for the 2DES spectra is set according to Fig. 1.

another. As can be seen, and upon comparison with the monomer spectrum given in Fig. 1, the fingerprints displayed essentially relate to those registered from their monomeric contributions, with the main $\mathrm{HL}^{2} \mathrm{ESA}$ in the $26-28 \mathrm{k} \mathrm{cm}^{-1}$ range, except for cluster $O$ which experiences a strong red-shift along both $\Omega_{1}$ and $\Omega_{3}$ due to the presence of a close-lying water molecule coordinating directly to the C4 position. No interaction is observed for clusters $\mathrm{H}$ and $\mathrm{Q}$, as indicated by the absence of the ${ }^{1} \mathrm{~L}_{\mathrm{a}}$ splitting that is a feature of the closer and more interacting clusters, despite the observation of slightly displaced $\mathrm{HL}^{2}$ signals along $\Omega_{3}$. Cluster $\mathrm{O}$, on the other hand, displays the shortest distance within this cluster group (5.09 $\AA$, see Fig. 6) and exhibits a slight splitting in the trace along $\Omega_{1}$, while showing no strong contributions in the low-energy Vis window. Despite possessing larger C5-C5 distances, clusters $\mathrm{H}$ and O exhibit CT ESA signals in the low energy $\left(\sim 15 \mathrm{k} \mathrm{cm}^{-1}\right)$ Vis probe window, which appear to be mediated mainly through the interaction of one of the amino groups with an aromatic group of the opposite adenine. While possessing large C5-C5 distances, the adenine moieties in $\mathrm{H}$ and $\mathrm{O}$ still remain at much closer amino-to-base distances, which are the distances facilitating the interaction and thus the reason behind the weak CT signals in the low-energy window. Therefore, the proximity of the amino groups to the aromatic moieties on the opposing base, also noted previously for cluster I in Fig. 5, appears to be an important aspect to consider for the appearance of CT states among adenine dimers like ApA.

The last group of clusters consists of those with the longest distances, beyond $6 \AA$, and comprises the clusters A, J and M (see Table 1), which amount to $16 \%$ of the sampled conformational space, and which contain the most repeated conformation (A) that accounts on its own for $10 \%$ of the overall population. Fig. 7 shows the different spectra, showcasing three apparently different behaviours. The most populated cluster A features joint ${ }^{1} \mathrm{~L}_{\mathrm{a}}$ traces along $\Omega_{1}$, and does not 


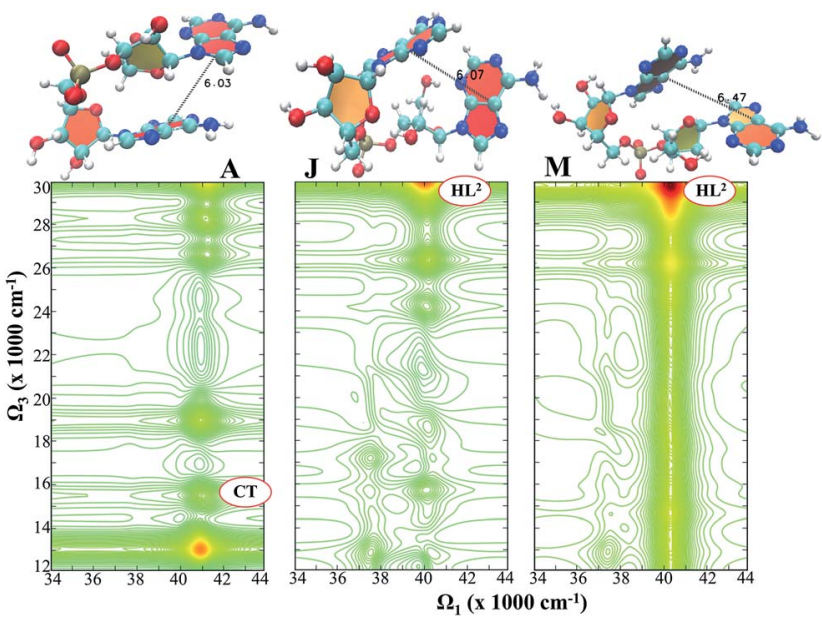

Fig. 7 2DES of the clusters with a C5-C5 distance of more than $6 \AA$ : A, J and M (see Table 1). The C5-C5 distances are shown on the structures. $\mathrm{HL}^{2}$ and $\mathrm{CT}$ labels are shown to aid in the assignment. The colour scheme for the 2DES spectra is set according to Fig. 1.

display the previously reported splitting due to strongly interacting $\pi$-stacked systems, while exhibiting a strong localised ESA at $\sim 13000$ and a CT signal at $\sim 15-16 \mathrm{k} \mathrm{cm}^{-1}$, with the more intense $\mathrm{HL}^{2}$ being placed in this case beyond the Vis probe window. The relative intensity of the CT state is attributed to the proximity of one of the adenine's amino groups to the 5-membered-ring of the opposing moiety. This interaction, which appears to stabilise CT transitions, is behind most T-stacked interactions, particularly those mediated by the interaction of the amino group with the opposing nucleobase, and is thus expected to be a fingerprint for these particular conformations, coupled with the lack of broadening along $\Omega_{1}$ which is characteristic of these distant nuclear arrangements. Cluster J shows a qualitatively different spectrum due to the partial appearance of the $\mathrm{HL}^{2}$ state in the high-energy window, which obscures the rest of the localised signals that appear in the lower energy window and displays no CT contributions. Cluster M, on the other hand, exhibits monomer-like ESAs, displaying a weak signal at $\sim 14000 \mathrm{~cm}^{-1}$ that is attributed to a localised ESA. Overall, more distant bases lack the additional broadening along $\Omega_{1}$, which arises due to the splitting of the two ${ }^{1} \mathrm{~L}_{\mathrm{a}}$ states of the different strongly interacting adenine moieties in ApA, while exhibiting a degree of charge transfer character in T-stacked-like conformations. This is particularly the case for those structures where the amino group points towards the opposing 5-ring moiety, thus making them fingerprints for these particular conformations coupled with narrow bands along $\Omega_{1}$. It is worth noting that both $\pi$ - and T-stacked CT signals appear in the same energy window, with the different expected broadenings along $\Omega_{1}$ again being the only way to disentangle them.

Unfortunately, no 2DES experimental data is currently available for ApA to compare with our present findings, with the first time-resolved 2D spectrum for water-solvated adenine in the UV-pump and probe windows being recently reported in the literature, ${ }^{17}$ together with a theoretical model rationalising the 
different signals exhibited and their monitoring. ${ }^{64}$ This high-energy window employed has been shown to be capable of registering the excited state dynamics of adenine also in 1D pump-probe spectroscopy by means of monitoring the GSB recovery. ${ }^{50}$ While capable of registering the dynamics of one single nucleobase, this window is expected to not be as efficient for monitoring dimeric ${ }^{7}$ and multimeric $^{65}$ species featuring a range of different conformations with very distinct associated decay lifetimes. In this sense, the present work proposes the UV-pump/ Vis-probe range as a more suitable spectral window for monitoring of the different conformations observed in multimeric species, displaying distinct fingerprints that may be associated with particular nuclear arrangements, albeit their experimental characterisation may prove more challenging. Upcoming experimental efforts, coupled with carefully built theoretical models, will be required in the near future in order to ascertain the role played by different conformations in the photophysics and photochemistry of dimeric and multimeric species like ApA.

\section{Conclusions}

A computational protocol is introduced that enables the simulation of 2DES of highly flexible solvated monophosphate dinucleosides and the disentangling of the spectroscopic fingerprints of various stacked/unstacked configurations of the ApA dimer case. The 2DES spectra are expected to reveal information about the molecular arrangements in dimeric and multimeric systems, thus going beyond the limitations of standard 1D pump-probe techniques for the study of photoinitiated processes. By employing ground state molecular dynamics and clustering techniques, 17 structures of the water-solvated ApA dimer have been selected in order to represent $\sim 75 \%$ of the sampled conformational space. Accurate QM/MM RASPT2 computations have been carried out on each of the selected structures to obtain the electronic energy levels and transition dipole moments in order to simulate the 2DES signals within the SOS//QM/MM approach. By weighting in the contributions of each cluster to the signal according to their relative populations, an overall 2DES spectrum is obtained, showing fingerprints relating to both unstacked (non-interacting) and either $\pi$ - or T-stacked (interacting) ApA conformations. The extracted clusters can be analysed one-by-one, thus enhancing our understanding of the nuclear arrangements behind specific spectral signals and allowing us to characterise particular fingerprints that arise for each different possible intermolecular interaction. We show how for $\pi$-stacked arrangements, a splitting of the two ${ }^{1} \mathrm{~L}_{\mathrm{a}}$ traces of both adenine moieties leads to a marked broadening of the ${ }^{1} \mathrm{~L}_{\mathrm{a}}$ trace along $\Omega_{1}$, which can potentially mark the appearance of strongly interacting stacked species, thus being a specific fingerprint of 2DES that cannot be observed by standard pumpprobe set-ups. T-stacked conformations, on the other hand, are shown to exhibit relatively intense ESA CT signals in the low-energy Vis window through the interaction between the amino group of one adenine monomer and the opposing base, and are particularly intense for those cases promoting amino to 5membered-ring interactions. Unstacked ApA conformations are shown to display very similar spectra to those of the monomer, as expected from these noninteracting species. Overall, 2DES is shown to be a promising alternative to standard pump-probe experiments for elucidating and studying the concrete 
photo-initiated events in DNA multimeric systems, being potentially able to separate contributions arising from a wide range of conformations and thus providing unique insights into how photostability and photo-damage is modulated due to the presence of extended and interacting nucleobase sequences in our genomic material.

\section{Acknowledgements}

M. G. acknowledges the support from the European Research Council Advanced Grant STRATUS (ERC-2011-AdG No. 291198). I. R. gratefully acknowledges the support from the Agence National de la Recherche (FEMTO-2DNA, ANR-15-CE290010) and ENS-Lyon (grants 900/S81/BS81-FR14 and MI-LOURD-FR15). J. S.-M. and I. R. acknowledge the use of the HPC resources of the "Pôle Scientifique de Modélisation Numérique" (PSMN) at ENS-Lyon, France. S. M. gratefully acknowledges the support of the National Science Foundation (grant CHE1663822), and the Chemical Sciences, Geosciences, and Biosciences division, Office of Basic Energy Sciences, Office of Science, U.S. Department of Energy, award DE-FG02-04ER15571.

\section{Notes and references}

1 G. D. Scholes, G. R. Fleming, L. X. Chen, A. Aspuru-Guzik, A. Buchleitner, D. F. Coker, G. S. Engel, R. van Grondelle, A. Ishizaki, D. M. Jonas, J. S. Lundeen, J. K. McCusker, S. Mukamel, J. P. Ogilvie, A. Olaya-Castro, M. A. Ratner, F. C. Spano, K. B. Whaley and X. Zhu, Nature, 2017, 543, 647-656.

2 C. E. Crespo-Hernandez, B. Cohen, P. M. Hare and B. Kohler, Chem. Rev., 2004, 104, 1977-2019.

3 C. T. Middleton, K. de La Harpe, C. Su, Y. K. Law, C. E. Crespo-Hernandez and B. Kohler, in Annual Review of Physical Chemistry, Annual Reviews, Palo Alto, 2009, vol. 60, pp. 217-239.

4 D. Markovitsi, T. Gustavsson and I. Vayá, J. Phys. Chem. Lett., 2010, 1, 32713276.

5 W. J. Schreier, P. Gilch and W. Zinth, Annu. Rev. Phys. Chem., 2015, 66, 497-519.

6 W. J. Schreier, T. E. Schrader, F. O. Koller, P. Gilch, C. E. Crespo-Hernández, V. N. Swaminathan, T. Carell, W. Zinth and B. Kohler, Science, 2007, 315, 625-629.

7 T. Takaya, C. Su, K. d. L. Harpe, C. E. Crespo-Hernández and B. Kohler, Proc. Natl. Acad. Sci. U. S. A., 2008, 105, 10285-10290.

8 A. Giussani, J. Segarra-Martí, D. Roca-Sanjuán and M. Merchán, in Photoinduced Phenomena in Nucleic Acids I, ed. M. Barbatti, A. C. Borin and S. Ullrich, Springer International Publishing, 2015, ch. 501, vol. 355, pp. 57-97.

9 M. Barbatti, A. J. A. Aquino, J. J. Szymczak, D. Nachtigallova, P. Hobza and H. Lischka, Proc. Natl. Acad. Sci. U. S. A., 2010, 107, 21453-21458.

10 L. Serrano-Andrés and M. Merchán, J. Photochem. Photobiol., C, 2009, 10, 2132.

11 D. B. Bucher, B. M. Pilles, T. Carell and W. Zinth, Proc. Natl. Acad. Sci. U. S. A., 2014, 111, 4369-4374.

12 D. B. Bucher, C. L. Kufner, A. Schlueter, T. Carell and W. Zinth, J. Am. Chem. Soc., 2016, 138, 186-190. 
13 L. E. Buchanan, J. K. Carr, A. M. Fluitt, A. J. Hoganson, S. D. Moran, J. J. de Pablo, J. L. Skinner and M. T. Zanni, Proc. Natl. Acad. Sci. U. S. A., 2014, 111, 5796-5801.

14 A. Ghosh, J.-J. Ho, A. L. Serrano, D. R. Skoff, T. Zhang and M. T. Zanni, Faraday Discuss., 2015, 177, 493-505.

15 B. A. West, J. M. Womick and A. M. Moran, J. Phys. Chem. A, 2011, 115, 86308637.

16 B. A. West and A. M. Moran, J. Phys. Chem. Lett., 2012, 3(18), 2575-2581.

17 V. I. Prokhorenko, A. Picchiotti, M. Pola, A. G. Dijkstra and R. J. D. Miller, J. Phys. Chem. Lett., 2016, 7, 4445-4450.

18 I. Rivalta, A. Nenov, G. Cerullo, S. Mukamel and M. Garavelli, Int. J. Quantum Chem., 2014, 114, 85-93.

19 S. Mukamel, Principles of Nonlinear Optical Spectroscopy, O.U.P., New York, 1995.

20 S. Mukamel, Annu. Rev. Phys. Chem., 2000, 51, 691-729.

21 D. Polli, I. Rivalta, A. Nenov, O. Weingart, M. Garavelli and G. Cerullo, Photochem. Photobiol. Sci., 2015, 14, 213-228.

22 Q. Li, A. Giussani, J. Segarra-Martí, A. Nenov, I. Rivalta, A. A. Voityuk, S. Mukamel, D. Roca-Sanjuán, M. Garavelli and L. Blancafort, Chem.-Eur. J., 2016, 22, 7497-7507.

23 A. Nenov, S. a. Beccara, I. Rivalta, G. Cerullo, S. Mukamel and M. Garavelli, ChemPhysChem, 2014, 15, 3282-3290.

24 D. Case, T. A. Darden, T. E. Cheatham, C. Simmerling, J. Wang, R. Duke, R. Luo, M. Crowley, R. Walker, W. Zhang, K. M. Merz, B. Wang, S. Hayik, A. Roitberg, G. Seabra, I. Kolossváry, K. F. Wong, F. Paesani, J. Vanicek, X. Wu, S. Brozell, T. Steinbrecher, H. Gohlke, L. Yang, C. Tan, J. Mongan, V. Hornak, G. Cui, D. H. Mathews, M. G. Seetin, C. Sagui, V. Babin and P. Kollman, Amber 11, University of California, San Francisco, 2011.

25 D. A. Case, T. E. Cheatham, T. Darden, H. Gohlke, R. Luo, K. M. Merz, A. Onufriev, C. Simmerling, B. Wang and R. J. Woods, J. Comput. Chem., 2005, 26, 1668-1688.

26 A. Pérez, I. Marchán, D. Svozil, J. Sponer, T. E. Cheatham III, C. A. Laughton and M. Orozco, Biophys. J., 2007, 92, 3817-3829.

27 W. L. Jorgensen, J. Chandrasekhar, J. D. Madura, R. W. Impey and M. L. Klein, J. Chem. Phys., 1983, 79, 926-935.

28 A. Nenov, A. Giussani, J. Segarra-Martí, V. K. Jaiswal, I. Rivalta, G. Cerullo, S. Mukamel and M. Garavelli, J. Chem. Phys., 2015, 142, 212443.

29 F. Aquilante, J. Autschbach, R. Carlson, L. Chibotaru, M. G. Delcey, L. De Vico, I. Fernández Galvan, N. Ferré, L. M. Frutos, L. Gagliardi, M. Garavelli, A. Giussani, C. Hoyer, G. Li Manni, H. Lischka, D. Ma, P. A. Malmqvist, T. Müller, A. Nenov, M. Olivucci, T. B. Pedersen, D. Peng, F. Plasser, B. Pritchard, M. Reiher, I. Rivalta, I. Schapiro, J. Segarra-Martí, M. Stenrup, D. G. Truhlar, L. Ungur, A. Valentini, S. Vancoillie, V. Veryazov, V. Vysotskiy, O. Weingart, F. Zapata and R. Lindh, J. Comput. Chem., 2016, 37, 506-541.

30 P. A. Malmqvist, A. Rendell and B. O. Roos, J. Phys. Chem., 1990, 94, 5477-5482. 31 P. Å. Malmqvist, K. Pierloot, A. R. M. Shahi, C. J. Cramer and L. Gagliardi, J. Chem. Phys., 2008, 128, 204109.

32 V. Saurí, L. Serrano-Andrés, A. R. M. Shahi, L. Gagliardi, S. Vancoillie and K. Pierloot, J. Chem. Theory Comput., 2011, 7, 153-168. 
33 F. Aquilante, R. Lindh and T. Bondo Pedersen, J. Chem. Phys., 2007, 127, 114107.

34 F. Aquilante, T. B. Pedersen and R. Lindh, J. Chem. Phys., 2007, 126, 194106.

35 T. Pedersen, F. Aquilante and R. Lindh, Theor. Chem. Acc., 2009, 124, 1-10.

36 P. O. Widmark, B. Joakim Persson and B. O. Roos, Theor. Chim. Acta, 1991, 79, 419-432.

37 S. F. Altavilla, J. Segarra-Martí, A. Nenov, I. Conti, I. Rivalta and M. Garavelli, Front. Chem., 2015, 3, 29.

38 N. Forsberg and P. A. Malmqvist, Chem. Phys. Lett., 1997, 274, 196-204.

39 J. P. Zobel, J. J. Nogueira and L. Gonzalez, Chem. Sci., 2017, 8, 1482-1499.

40 G. Ghigo, B. O. Roos and P.-Å. Malmqvist, Chem. Phys. Lett., 2004, 396, 142149.

41 P.-Å. Malmqvist and B. O. Roos, Chem. Phys. Lett., 1989, 155, 189-194.

42 P. Å. Malmqvist, B. O. Roos and B. Schimmelpfennig, Chem. Phys. Lett., 2002, 357, 230-240.

43 S. Mukamel, R. Oszwaldowski and D. Abramavicius, Phys. Rev. B: Condens. Matter Mater. Phys., 2007, 75, 245305.

44 D. Abramavicius, B. Palmieri, D. V. Voronine, F. Šanda and S. Mukamel, Chem. Rev., 2009, 109, 2350-2408.

45 M. Richter and B. P. Fingerhut, J. Chem. Theory Comput., 2016, 12, 3284-3294.

46 A. Nenov, A. Giussani, B. P. Fingerhut, I. Rivalta, E. Dumont, S. Mukamel and M. Garavelli, Phys. Chem. Chem. Phys., 2015, 17, 30925-30936.

47 R. Tempelaar, C. P. van der Vegte, J. Knoester and T. L. C. Jansen, J. Chem. Phys., 2013, 138, 164106.

48 C. P. van der Vegte, A. G. Dijkstra, J. Knoester and T. L. C. Jansen, J. Phys. Chem. A, 2013, 117, 5970-5980.

49 M. Kowalewski, B. P. Fingerhut, K. E. Dorfman, K. Bennett and S. Mukamel, Chem. Rev., 2017, 117(19), 12165-12226.

50 B. Cohen, P. M. Hare and B. Kohler, J. Am. Chem. Soc., 2003, 125, 13594-13601.

51 T. Gustavsson, A. Sharonov, D. Onidas and D. Markovitsi, Chem. Phys. Lett., 2002, 356, 49-54.

52 F. Santoro, R. Improta, T. Fahleson, J. Kauczor, P. Norman and S. Coriani, J. Phys. Chem. Lett., 2014, 5, 1806-1811.

53 P. G. Szalay, T. Watson, A. Perera, V. F. Lotrich and R. J. Bartlett, J. Phys. Chem. A, 2012, 116, 6702-6710.

54 R. Improta and V. Barone, Excited States Behavior of Nucleobases in Solution: Insights from Computational Studies, in Photoinduced Phenomena in Nucleic Acids I. Topics in Current Chemistry, ed. M. Barbatti, A. Borin and S. Ullrich, Springer, 2014, vol. 355, pp. 329-357.

55 A. J. Pepino, J. Segarra-Martí, A. Nenov, R. Improta and M. Garavelli, J. Phys. Chem. Lett., 2017, 8, 1777-1783.

56 L. Martinez-Fernandez, A. J. Pepino, J. Segarra-Martí, J. Jovaisaite, I. Vayá, A. Nenov, D. Markovitsi, T. Gustavsson, A. Banyasz, M. Garavelli and R. Improta, J. Am. Chem. Soc., 2017, 139, 7780-7791.

57 A. Giussani, J. Segarra-Martí, A. Nenov, I. Rivalta, A. Tolomelli, S. Mukamel and M. Garavelli, Theor. Chem. Acc., 2016, 135, 1-18.

58 S. Jafilan, L. Klein, C. Hyun and J. Florián, J. Phys. Chem. B, 2012, 116, 36133618. 
59 I. Stiopkin, T. Brixner, M. Yang and G. R. Fleming, J. Phys. Chem. B, 2006, 110, 20032-20037.

60 N. S. Ginsberg, Y.-C. Cheng and G. R. Fleming, Acc. Chem. Res., 2009, 42, 13521363.

61 I. Conti, A. Nenov, S. Ho, S. F. Altavilla, I. Rivalta, E. Dumont, G. Orlandi and M. Garavelli, Phys. Chem. Chem. Phys., 2015, 17, 7291-7302.

62 A. Nenov, J. Segarra-Martí, A. Giussani, I. Conti, I. Rivalta, E. Dumont, V. K. Jaiswal, S. F. Altavilla, S. Mukamel and M. Garavelli, Faraday Discuss., 2015, 177, 345-362.

63 L. Martínez-Fernández, A. J. Pepino, J. Segarra-Martí, A. Banyasz, M. Garavelli and R. Improta, J. Chem. Theory Comput., 2016, 12, 4430-4439.

64 A. G. Dijkstra and V. I. Prokhorenko, J. Chem. Phys., 2017, 147, 064102.

65 C. Su, C. T. Middleton and B. Kohler, J. Phys. Chem. B, 2012, 116, 10266-10274. 